\title{
Nasal Colonization and Antimicrobial Susceptibility Pattern of Staphylococcus Species among Children in Lahore, Pakistan
}

\author{
Amtul Jamil Sami ${ }^{*}$, Madeeha Khalid ${ }^{1}$, Shajia Jabeen ${ }^{1}$, Sharqa Khaliil, Ghazala Sajjad ${ }^{1}$, Noor-ul-Ain ${ }^{1}$, Rabia \\ Arooj $^{1}$ \\ ${ }^{1}$ Institute of Biochemistry and Biotechnology, University of the Punjab, Lahore, Pakistan
}

Received May 28, 2018; Accepted Nov. 05, 2018

\begin{abstract}
Introduction: Staphylococcus is a genus of pathogenic bacteria, which asymptomatically colonizes the upper respiratory tract of the human. The incidence of invasive Staphylococcal infections and the disease burden are high among children in South Asia, including Pakistan. This study aims to determine the nasal colonization and antimicrobial susceptibility pattern of Staphylococcus species isolated from preschool children in Lahore, Pakistan. Methods: A community-based study was conducted in two camps named Shah Di Khui and Jeevan Haana in Lahore city. A total of 100 nasal samples, were collected from preschool children from lower-middle-class families during January to March 2018. Species identification was performed using the coagulase test, catalase test, and Gram staining. Also, a $370 \mathrm{bp}$ fragment of the $t u f$ gene was targetted using specific primers for the genus Staphylococcus. Antibiotic resistance pattern of the isolates was defined by an antibiotic susceptibility test using a series of antibiotic discs. Results: The results of this study indicated the presence of Staphylococcus species, mainly Staphylococcus aureus in more than $85 \%$ of the children. PCR amplification of tuf gene confirmed the identity of the $S$. aureus isolates from the nasal cultures. Many showed resistance to more than two broad-spectrum antibiotics. Conclusion: The prevalence of nasal colonization of $S$. aureus was more than $85 \%$ among preschool children. Most of the isolates were resistant to $\beta$-lactam antibiotics. J Med Microbiol Infec Dis, 2018, 6 (4): 91-98.
\end{abstract}

Keywords: Staphylococcus, Drug resistance, Invasive burden, MRSA.

\section{INTRODUCTION}

The human body contains numerous microorganisms, the so-called microbiota that outnumbers human cells. The human nasal passage is one of the primary habitats for microflora as well as pathogenic agents. The nasal passage of the human nostrils leads to the nasopharynx and the upper back part of the throat. The microbial communities inhabiting the nasal passages of humans colonize soon after the birth and distinctly changes over the lifespan of an individual with high inter-individual variations. During a human lifetime, the bacterial colonization of the human nasal cavities and its variations are affected by various factors, including the development of the immunity, hormonal changes, and age. Other affecting factors include environmental variations, e.g., temperature, humidity, pollution, and airborne microbes [1].

In children, a very complex community of bacteria inhabit the nasal passages. Identification of composition and dynamics of these bacteria can provide more insights into the basis of respiratory diseases [2]. Many works have demonstrated that the structure of pediatric bacterial microbiota of the nose is affected by acute respiratory tract infections (ARIs). Some studies have also reported that bacterial flora of the nasal cavities plays a vital role in regulating various immune responses in humans [3]. The interaction between bacterial species in nasal passages and the host include mutualism, commensalism, and pathogenic associations [4]. The most common species of nasal microflora comprise Staphylococcus aureus,
Staphylococcus epidermidis, Streptococcus pneumoniae, Micrococcus luteus, Haemophilus influenzae, Proteus vulgaris, Proteus mirabilis, and Bacillus sp. [2, 3].

The members of the genus Staphylococcus are among the common bacteria inhabiting the nasal passages of children and $S$. aureus, S. epidermidis, and S. hominis are among the most prevalent species. These Gram-positive bacteria are catalase positive, non-motile, and facultative anaerobes. They cause acute to severe infections, such as serious skin infections, pimples, boils, carditis, meningitis, septicemia, arthritis, endocarditis, abscess, osteomyelitis, central venous catheter-associated bacteremia, pneumonia, and ventilator-associated pneumonia. These bacteria produce the enzyme coagulase and are characterized as pathogenic or relatively pathogenic bacteria [5].

Pathogenic strains of the genus Staphylococcus such as $S$. aureus are usually coagulase-positive. The coagulasenegative strains, e.g., S. epidermidis, are mostly less invasive.

However, they are progressively considered pathogens

*Correspondence: Amtul Jamil Sami

Institute of Biochemistry and Biotechnology, University of the Punjab, Lahore, Pakistan, 54590.

Email: amtuljamilsami@yahoo.com

Tel: +92 3327845179 Fax: +92 4235220855 
as they may cause nosocomial infections [6-7].

The bacteria $S$. aureus can pathogenically or nonpathogenically colonize humans [8]. It colonizes the nasal passages in about $33 \%$ of humans and has emerged as a significant antibiotic-resistant bacterium since 1960 [9]. One of the major underlying cause of antibiotic-resistance development is the inappropriate use of these medications. Apart from the inherent ability of microbes, agents, various factors including overuse and lack of access to appropriate combinations as well as failure to complete treatment regimens are involved in development of resistance. Moreover, technological advances and ease of travel contribute to the global spread of antibiotic resistance [1].

Resistance to almost all classes of antibiotics has been reported in different strains of Staphylococcus species. The DNA analysis of various strains of $S$. aureus has revealed the presence of virulence factors in these bacteria, explaining their pathogenic and invasive nature [10]. In addition to the presence of virulence factors and toxic genes, the occurrence of mutations in chromosomal and plasmid DNA is common and widespread in Staphylococcus species.

The antibiotics families macrolides, lincosamides, and streptogramins (introduced in 1952) kills the bacteria by targeting the bacterial 50S ribosomal subunit and inhibiting protein synthesis. However, shortly after their introduction for the treatment of staphylococcal infections, resistance, mainly among $S$. aureus strains, was observed. In this species, ermA, ermB, or erm $C$ genes, located on plasmids or chromosomes, are responsible for ribosomal modification and making the antibiotics ineffective. Also, aminoglycoside resistance in Staphylococcus species is due to a chromosomal modification, leading to altered binding sites of this combination, i.e., ribosomes [11]. Fluoroquinolones, initially introduced to treat Gramnegative infections, were also effective against some Grampositive species including staphylococci through inhibition of bacterial DNA gyrase. Resistance against these combinations rapidly emerged as mutations developed in bacterial DNA gyrase gene. Resistance to $\beta$-lactam antibiotics is the most widespread antibacterial resistance in staphylococci, mainly $S$. aureus and $S$. epidermidis. The $\beta$ lactams are a family of broad-spectrum antibiotics including cephems (cephalosporins), penam-penicillin derivatives (methicillin, oxacillin, nafcillin, ampicillin), and carbapenems. [12]. The strains resistant to these antibiotics are classified as methicillin-resistant bacteria, e.g., methicillin-resistant $S$. aureus (MRSA) and methicillinresistant $S$. epidermidis (MRSE). The emergence of multiantibiotic resistant strains, such as MRSA has made the treatment of the infections much more difficult [13].

As mentioned above, the environmental factors and climatic conditions play a major role in the development of the microbial flora. In Pakistan, climatic conditions show a high variation in different regions of the country, and in some parts of the country, the weather is hot and humid most time of the year. The variation of weather conditions contributes to the prevalence of different microbial flora, including Staphylococcus species. There is no regional study on the incidence and burden of invasive staphylococcal species in children. There is also data on nasal colonization of Staphylococcus among pre-school children in Pakistan. This study aims to investigate the nasal colonization and antimicrobial susceptibility pattern of Staphylococcus species among children $\leq$ five years of age in Lahore, Pakistan. The results of the study will be helpful in the diagnosis and eradication of Staphylococcusinduced pneumonia, the primary deadly disease in children of Pakistan.

\section{MATERIAL AND METHODS}

Collection of nasal samples. Two camps, Shah Di Khui (near Punjab University) and Jeevan Haana (near Barkat market) in Lahore Pakistan were selected. The nasal secretions were collected by sterilized swabs from healthy children $\leq 5$ years of age belonging to lower and middleclass families. The swabs were individually placed in the sterile tubes and transported to the laboratory.

The consent for the collection of specimens was obtained from childrens' parents. The study was approved by Higher Education of Pakistan S.No NRPU 4269.

Preparation of STGG transport medium. The skim milk-tryptone-glucose-glycerol (STGG) transport medium was prepared for inoculation and culture of nasal samples. The media was prepared by skim milk powder $(2 \%)$, tryptone soy broth (TSB), $(3 \%)$, glucose $(0.5 \%)$, and $10 \%$ glycerol in distilled water. The media were sterilized and stored at $4^{\circ} \mathrm{C}$ until use.

Inoculation of STGG with nasopharyngeal swabs. The nasal samples were inoculated into vials containing 3 $\mathrm{ml}$ of STGG medium followed by overnight incubation in a $120 \mathrm{rpm}$ shaking incubator at $37^{\circ} \mathrm{C}$. The cultures were checked after 18 hours for bacterial growth.

Culture. Blood agar medium was prepared by dissolving blood agar base in distilled water followed by autoclaving. Once, the culture media cooled to $50{ }^{\circ} \mathrm{C}, 5 \%$ chicken blood was added to them. Amounts of $20 \mathrm{ml}$ of the blood agar were dispensed into Petri dishes and allowed to cool and solidify, and then, sealed with parafilm and stored at $4^{\circ} \mathrm{C}$. The plates were streaked by previously cultured nasal samples using the standard streaking technique, and sealed with parafilm and incubated at $37^{\circ} \mathrm{C}$ overnight. The following day, the plates were examined for bacterial growth.

Identification of Staphylococcus species. The Staphylococcus colonies appeared as round, creamy white colonies. The plates were also checked for beta hemolysis, which is the result of alpha-toxin production by Staphylococcus species. Further identification of the bacteria was performed by using standard Gram staining technique and coagulase, catalase, oxidase, and indole tests. For the catalase test, a bacterial colony was placed on a slide and mixed with a drop of distilled water to form a white suspension and then a drop of $3 \%$ hydrogen peroxide solution was added to it. The rapid evolution of $\mathrm{O}_{2}$ along with bubble formation indicated a positive result.

For coagulase test, a bacteria colony was emulsified in a drop of water on a clean glass slide, and then some 
undiluted plasma was picked with a sterilized loop and mixed with the milky solution of the bacterial colony. The clumping of cocci, visible to naked eye within 10 seconds, revealed coagulase positive isolates. For indole assay, the bacteria were grown in tryptic soy broth in 10-ml culture tubes for 24 hours. The following day, few drops of Kovac's reagent (isoamyl alcohol, paradimethylaminobenzaldehyde (DMAB), and concentrated $\mathrm{HCl}$ ), were added into the tube. Formation of a red ring at the interface indicated a positive indole result.
Oxidase test was performed using filter papers soaked with tetramethyl-p-phenylenediamine dihydrochloride substrate. The filter paper was moistened with sterile water, and a bacteria was smeared on the filter paper. Color change to purple or deep blue within 10-30 seconds indicated a positive oxidase result [14].

Identification of $S$. aureus by PCR. The identity of Staphylococcus spp. was confirmed by amplification of the tuf gene as described by others [15].

Table 1. The primers used in this study

\begin{tabular}{l|lll}
\hline \multicolumn{1}{c}{ Primer } & Name & Sequence & The binding region on the gene \\
\hline $\begin{array}{l}\text { Forward primer } \\
\text { Reverse primer }\end{array}$ & TStaG422 & 5'-GGC CGT GTT GAA CGT GGT CAA ATC A-3' & $422-446 *$ \\
\hline
\end{tabular}

*The nucleotide positions are given with reference to the $t u f$ gene sequence of $E$. coli (Accession no: J01690)

PCR amplification was performed in a $25 \mu \mathrm{l}$ reactions containing $5 \mu \mathrm{l}$ of microbial culture, $0.5 \mu \mathrm{M}$ of forward and reverse primers (Table 1), and $12.5 \mu \mathrm{l}$ of GoTaq Green Master Mix, (Promega, Madison, WI, USA), and nucleasefree water.

Antibiotic susceptibility assay. Bacteria susceptibility assays were performed by disc diffusion method according to Clinical and Laboratory Standards Institute (CLSI) reference methods [16]. For examination of the susceptibility pattern of the isolates, antimicrobial testing was performed by using standardized Kirby-Bauer disc diffusion test $[17,18]$ on $10 \mathrm{~mm}$ Mueller-Hinton agar plates ( $\mathrm{pH}$ 7.2-7.4). The bacteria density of isolates in suspensions was adjusted to $0.5 \mathrm{McF}$ arland standard. Amounts of $2 \mathrm{ml}$ saline solution were prepared for each sample, and colonies from blood agar plates were suspended in salt solution. The prepared suspensions were then streaked 2-3 times onto Mueller-Hinton agar plates. The antibiotic susceptibility testing (including MRSA screening with cefoxitin as the recommended by NCCLS) [16], was performed using the the antibiotic discs, cefoxitin $(30 \mu \mathrm{g})$, trimethoprim/ sulfamethoxazole $\quad(25 \mu \mathrm{g}), \quad$ ciprofloxacin $\quad(5 \mu \mathrm{g})$, erythromycin $(15 \mu \mathrm{g})$, amikacin $(30 \mu \mathrm{g})$, penicillin (10 units), novobiocin $(30 \mu \mathrm{g})$, and vancomycin $(30 \mu \mathrm{g})$ (Thermo Fisher Scientific, Hampshire, England). The plates were incubated at $37^{\circ} \mathrm{C}$ for $18-24 \mathrm{~h}$ and then were examined for bacterial growth. The clear zone of inhibition around each disk was measured by calipers.

\section{RESULTS}

Microflora. The cultures revealed different species of pathogenic microorganisms, including S. aureus, Bacillus spp., Proteus mirabilis, Staphylococcus epidermidis, Acinetobacter spp., Haemophilus spp., Proteus vulgaris, and Macrococcus species. The presence of Staphylococcus species was confirmed by different biochemical tests, including catalase, coagulase, oxidase, and indole tests as well as Gram staining. The species $S$. aureus was the most prevalent bacteria in the nasal samples with a prevalent rate of over $85 \%$. Other species of bacteria and yeast cells were also identified in the nasal cavities of the children (Fig. 1).

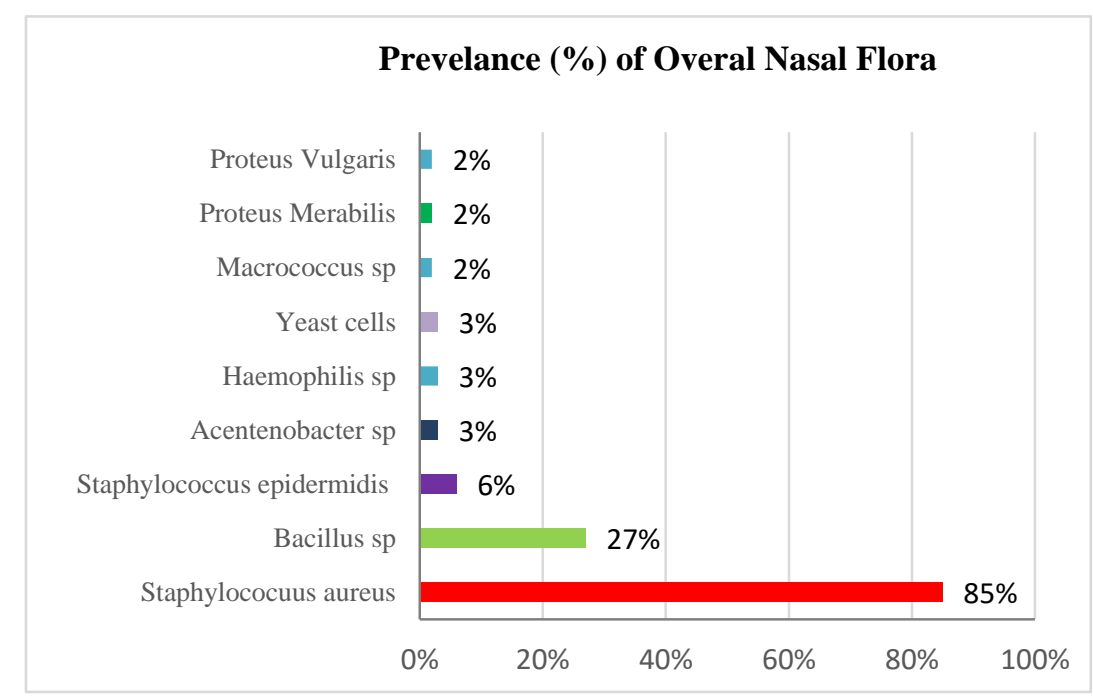

Fig. 1. Prevalence of pathogenic microbes in the nasal samples of children in Lahore, Pakistan 
PCR Assay for $S$. aureus. The species $S$. aureus was identified by PCR amplification of a $370 \mathrm{bp}$ fragment of the tuf gene specific to this species (Fig. 2).

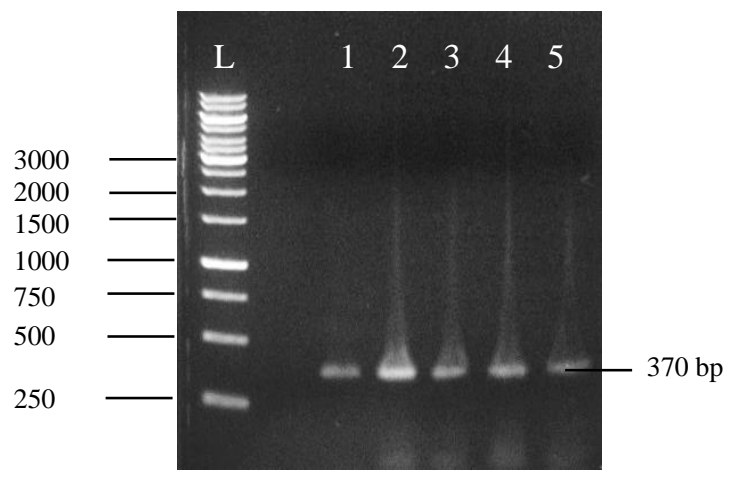

Fig. 2. PCR amplification of a $370 \mathrm{bp}$ fragment of tuf gene specific to $S$. aureus. Lane L, DNA ladder; lanes 1-5 positive samples

Susceptibility pattern of Staphylococcus species. Different combinations of drugs were used to identify resistance and susceptibility pattern of the Staphylococcal species. The isolates exhibited the highest resistance rates to penicillin, erythromycin, trimethoprim/sulfamethoxazole, and ciprofloxacin (Table 2).

Sensitivity analysis of Staphylococcus species to antibiotics. In our study, most isolates were found to be resistant to more than 2 antibiotics. The bacteria species in the nasal samples showed the highest resistance to penicillin $(98.90 \%)$ followed by erythromycin $(81.32 \%)$, trimethoprim/sulfamethoxazole $\quad(79.12 \%)$, amikacin
(41.6\%), ciprofloxacin (38.46\%), cefoxitin (10.99\%), and Novobiocin $(3.30 \%)$. All the species of microbes were found to be sensitive to vancomycin with $0 \%$ resistance (Fig. 3). The number of resistant and susceptible isolates of $S$. aureus and S. epidermidis to the tested antibiotics are shown in the Figure 4.

\section{DISCUSSION}

Nasal colonization of Staphylococcus species is a risk factor for the development of lung diseases. Children are considered as the persistent carriers of nasal pathogens with the highest rate of the carriage. The role of nasal carriage in $S$. aureus infections has been extensively studied and reported worldwide [19]. The nasal carriage and prevalence of $S$. aureus vary in children with socioeconomic status, general health, and disease conditions [20]. In Japan, 17.5\% of nasopharyngeal colonization of respiratory bacterial pathogens was observed among children attending day-care centers [21]. In India, nasal carriage of $S$. aureus among healthy preschool children of Ujjain was 6.3\%, from of which $16.3 \%$ were methicillin-resistant (MRSA) isolates [22]. In Ghana, 22.1\% of children showed colonization with $S$. aureus, with the highest carriage rates during the rainy seasons [23]. In Brazil, the highest colonization rates with $S$. aureus was reported among children $(48 \%)$ with $6.2 \%$ identified as MRSA [23, 24]. The identity of Staphylococcus species in our study was confirmed by the PCR amplification of tuf gene, a $370 \mathrm{bp}$ sequence specific to Staphylococcus genome (Fig. 2). The tuf gene encodes elongation factor tu (EF-tu), which is required for the peptide chain formation [15].

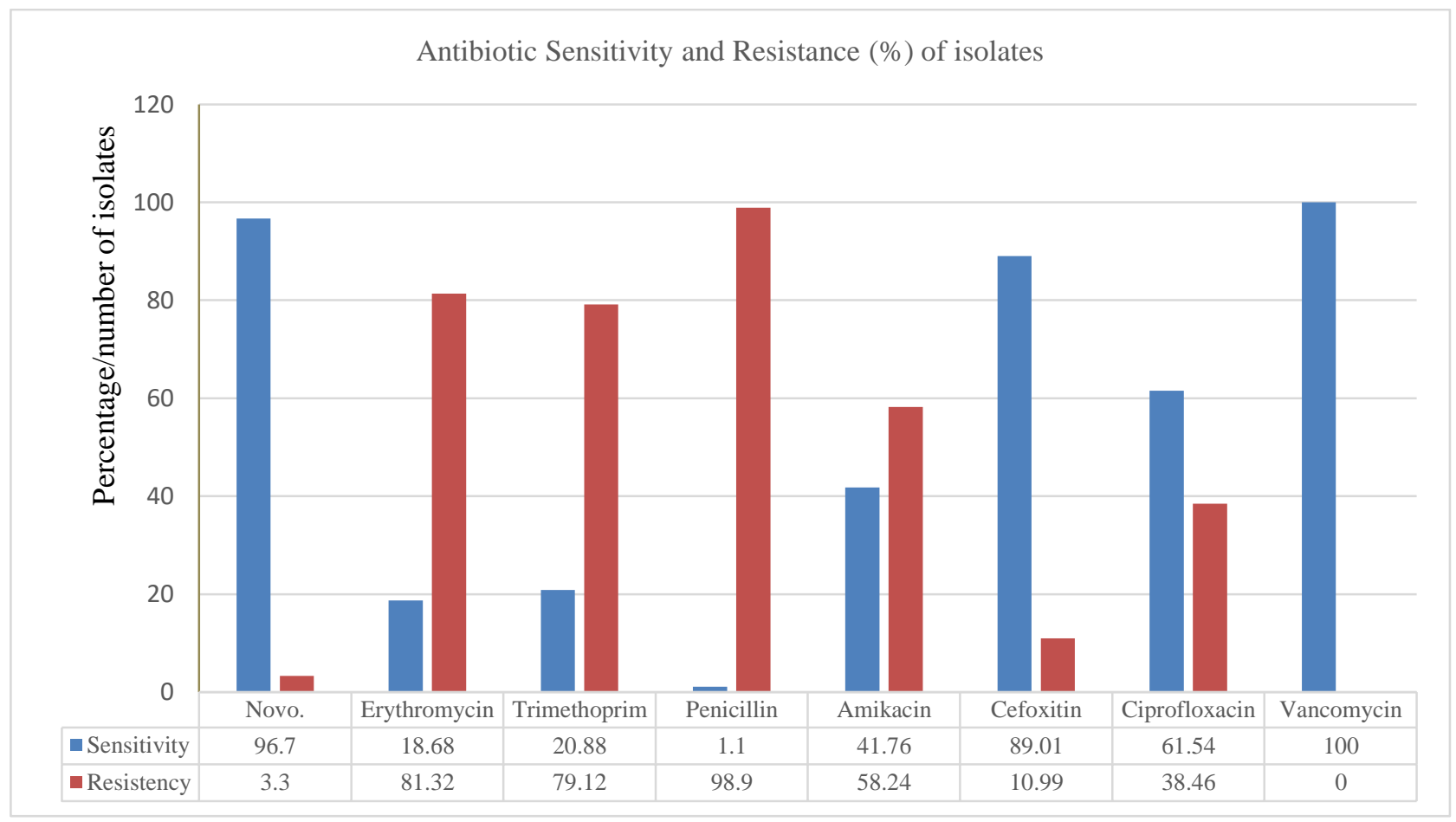

Fig. 3. Susceptibility pattern of Staphylococcus species in children under 5 years 
Table 2. Susceptibility of $S$. aureus and S. epidermidis to the tested antibiotics

\begin{tabular}{|c|c|c|c|c|c|}
\hline \multirow{2}{*}{$\begin{array}{l}\text { S. } \\
\text { No }\end{array}$} & \multirow[t]{2}{*}{ Antibiotics } & \multirow{2}{*}{$\begin{array}{l}\text { Total } \\
\text { samples }\end{array}$} & \multicolumn{2}{|c|}{ Species } & \multirow{2}{*}{$\begin{array}{l}\text { MRSA/ } \\
M R S E\end{array}$} \\
\hline & & & $\begin{array}{l}\text { S. } \\
\text { aureus }\end{array}$ & $\begin{array}{l}S . \\
\text { epidermidis }\end{array}$ & \\
\hline \multirow[t]{6}{*}{1} & Erythromycin & \multirow[t]{6}{*}{80} & 52 & 04 & $6 / 2$ \\
\hline & Trimethoprim/Sulfamethoxazole & & 63 & 01 & $6 / 2$ \\
\hline & Penicillin & & 76 & 04 & $8 / 2$ \\
\hline & Cefoxitin & & 0 & 0 & $8 / 2$ \\
\hline & Ciprofloxacin & & 27 & 02 & $5 / 1$ \\
\hline & Vancomycin & & 0 & 0 & $0 / 0$ \\
\hline \multirow[t]{15}{*}{2} & Penicillin and Ciprofloxacin & 71 & 27 & 02 & $4 / 1$ \\
\hline & Ciprofloxacin and Trimethoprim/Sulfamethoxazole & & 24 & 01 & $3 / 1$ \\
\hline & Vancomycin and Erythromycin & & 0 & 0 & $0 / 0$ \\
\hline & Cefoxitin and Vancomycin & & 0 & 0 & $0 / 0$ \\
\hline & Penicillin and Vancomycin & & 0 & 0 & $0 / 0$ \\
\hline & Erythromycin and Penicillin & & 62 & 04 & $6 / 2$ \\
\hline & Trimethoprim/Sulfamethoxazole and Cefoxitin & & 06 & 02 & $6 / 2$ \\
\hline & Trimethoprim/Sulfamethoxazole and Erythromycin & & 51 & 01 & $5 / 2$ \\
\hline & Trimethoprim/Sulfamethoxazole and Penicillin & & 68 & 03 & $6 / 2$ \\
\hline & Trimethoprim/Sulfamethoxazole and Vancomycin & & 0 & 0 & $0 / 0$ \\
\hline & Penicillin and Cefoxitin & & 08 & 02 & $8 / 2$ \\
\hline & Erythromycin and Cefoxitin & & 06 & 02 & $2 / 2$ \\
\hline & Erythromycin and Ciprofloxacin & & 28 & 03 & $3 / 1$ \\
\hline & Vancomycin and Ciprofloxacin & & 0 & 0 & $0 / 0$ \\
\hline & Cefoxitin and Ciprofloxacin & & 05 & 01 & $5 / 1$ \\
\hline 3 & Penicillin, Ciprofloxacin, and Erythromycin & 61 & 23 & 02 & $4 / 1$ \\
\hline & Vancomycin, Erythromycin and Trimethoprim/Sulfamethoxazole & & 0 & 0 & $0 / 0$ \\
\hline & Trimethoprim/Sulfamethoxazole, Penicillin, and Cefoxitin & & 06 & 02 & $6 / 2$ \\
\hline & Cefoxitin, Trimethoprim/Sulfamethoxazole, and Ciprofloxacin & & 3 & 01 & $3 / 1$ \\
\hline & Vancomycin, Ciprofloxacin, and Penicillin & & 0 & 0 & $0 / 0$ \\
\hline & Erythromycin, Penicillin and Trimethoprim/Sulfamethoxazole & & 51 & 2 & $6 / 2$ \\
\hline & Cefoxitin, Erythromycin, and vancomycin & & 0 & 0 & $0 / 0$ \\
\hline & Trimethoprim/ Sulfamethoxazole, Penicillin, and Erythromycin & & 58 & 03 & $5 / 2$ \\
\hline & Trimethoprim/ Sulfamethoxazole, Penicillin, and Vancomycin & & 0 & 0 & $0 / 0$ \\
\hline & Trimethoprim/ Sulfamethoxazole, Penicillin, and Cefoxitin & & 07 & 02 & $5 / 2$ \\
\hline & Trimethoprim/ Sulfamethoxazole, Penicillin, and Ciprofloxacin & & 26 & 01 & $4 / 1$ \\
\hline & Trimethoprim/ Sulfamethoxazole, Erythromycin, and Vancomycin & & 0 & 0 & $0 / 0$ \\
\hline & Trimethoprim/ Sulfamethoxazole, Erythromycin, and Cefoxitin & & 07 & 02 & $5 / 2$ \\
\hline & Trimethoprim/ Sulfamethoxazole, Erythromycin, and Ciprofloxacin & & 23 & 02 & $2 / 1$ \\
\hline & Trimethoprim/ Sulfamethoxazole, Cefoxitin, and Ciprofloxacin & & 05 & 01 & $5 / 1$ \\
\hline & Trimethoprim/ Sulfamethoxazole, Vancomycin, and Ciprofloxacin & & 0 & 0 & $0 / 0$ \\
\hline & Trimethoprim/ Sulfamethoxazole, Vancomycin, and Cefoxitin & & 0 & 0 & $0 / 0$ \\
\hline & Penicillin, Erythromycin, and Vancomycin & & 0 & 0 & $0 / 0$ \\
\hline & Penicillin, Erythromycin, and Cefoxitin & & 06 & 02 & $6 / 2$ \\
\hline & Penicillin, Erythromycin, and Ciprofloxacin & & 23 & 02 & $2 / 1$ \\
\hline & Penicillin, Vancomycin, and Cefoxitin & & 0 & 0 & $0 / 0$ \\
\hline & Penicillin, Vancomycin, and Ciprofloxacin & & 0 & 0 & $0 / 0$ \\
\hline & Penicillin, Cefoxitin, and Ciprofloxacin & & 0 & 0 & $0 / 0$ \\
\hline & Erythromycin, Vancomycin, and Cefoxitin & & 0 & 0 & $0 / 0$ \\
\hline & Erythromycin, Vancomycin, Ciprofloxacin & & 0 & 0 & $0 / 0$ \\
\hline & Vancomycin, Ciprofloxacin, and Cefoxitin & & 0 & 0 & $0 / 0$ \\
\hline & Erythromycin, Cefoxitin, and Ciprofloxacin & & 03 & 01 & $3 / 1$ \\
\hline 4 & Trimethoprim/Sulfamethoxazole, Penicillin, Erythromycin, Cefoxitin & 23 & 05 & 02 & $4 / 2$ \\
\hline & Trimethoprim/Sulfamethoxazole, Erythromycin, Vancomycin, Cefoxitin & & 0 & 0 & $0 / 0$ \\
\hline & Penicillin, Erythromycin, Vancomycin, Cefoxitin & & 0 & 0 & $0 / 0$ \\
\hline & Trimethoprim/Sulfamethoxazole, Penicillin, Vancomycin, Cefoxitin & & 0 & 0 & $0 / 0$ \\
\hline & Penicillin, Erythromycin, Vancomycin, Ciprofloxacin & & 0 & 0 & $0 / 0$ \\
\hline & Penicillin, Erythromycin, Cefoxitin, Ciprofloxacin & & 03 & 01 & $3 / 1$ \\
\hline & Erythromycin, Vancomycin, Cefoxitin, Ciprofloxacin & & 0 & 0 & $0 / 0$ \\
\hline & Trimethoprim/Sulfamethoxazole, Vancomycin, Cefoxitin, Ciprofloxacin & & 0 & 0 & $0 / 0$ \\
\hline & Trimethoprim/Sulfamethoxazole, Erythromycin, Cefoxitin, Ciprofloxacin & & 02 & 0 & $2 / 0$ \\
\hline & Trimethoprim/Sulfamethoxazole, Penicillin, Vancomycin, Ciprofloxacin & & 0 & 0 & $0 / 0$ \\
\hline & Trimethoprim/Sulfamethoxazole, Penicillin, Erythromycin, Vancomycin & & 0 & 0 & $0 / 0$ \\
\hline & Cefoxitin, Trimethoprim/Sulfamethoxazole, Ciprofloxacin, Penicillin & & 0 & 0 & $0 / 0$ \\
\hline & Vancomycin, Ciprofloxacin, Penicillin, Cefoxitin & & 0 & 0 & $0 / 0$ \\
\hline & Vancomycin Trimethoprim/Sulfamethoxazole, Cefoxitin Ciprofloxacin & & 0 & 0 & $0 / 0$ \\
\hline & Erythromycin, Penicillin Trimethoprim/Sulfamethoxazole, Ciprofloxacin & & 22 & 01 & $2 / 1$ \\
\hline 5 & Trimethoprim/Sulfamethoxazole, Penicillin, Erythromycin, Vancomycin, Ciprofloxacin & 03 & 0 & 0 & $0 / 0$ \\
\hline & Trimethoprim/Sulfamethoxazole, Penicillin, Erythromycin, Vancomycin, Cefoxitin & & 0 & 0 & $0 / 0$ \\
\hline & Penicillin, Erythromycin, Vancomycin, Cefoxitin, Ciprofloxacin & & 0 & 0 & $0 / 0$ \\
\hline & Trimethoprim/Sulfamethoxazole, Erythromycin, Vancomycin, Cefoxitin, Ciprofloxacin & & 0 & 0 & $0 / 0$ \\
\hline & Trimethoprim/Sulfamethoxazole, Penicillin, Vancomycin, Cefoxitin, Ciprofloxacin & & 0 & 0 & $0 / 0$ \\
\hline & Trimethoprim/Sulfamethoxazole, Penicillin, Erythromycin, Cefoxitin, Ciprofloxacin & & 02 & 01 & $2 / 1$ \\
\hline & Erythromycin, Vancomycin, Trimethoprim/Sulfamethoxazole, Cefoxitin Ciprofloxacin, Penicillin & & 0 & 0 & $0 / 0$ \\
\hline & Vancomycin, Erythromycin, Trimethoprim/Sulfamethoxazole, Cefoxitin Ciprofloxacin, Penicillin & & 0 & 0 & $0 / 0$ \\
\hline & Trimethoprim/Sulfamethoxazole, Cefoxitin, Ciprofloxacin, Penicillin Vancomycin, Erythromycin & & 0 & 0 & $0 / 0$ \\
\hline & Cefoxitin, Ciprofloxacin, Penicillin, Vancomycin, Trimethoprim/Sulfamethoxazole, Erythromycin & & 0 & 0 & $0 / 0$ \\
\hline & Ciprofloxacin, Cefoxitin, Penicillin, Vancomycin, Trimethoprim/Sulfamethoxazole, Erythromycin & & 0 & 0 & $0 / 0$ \\
\hline & Penicillin, Cefoxitin, Ciprofloxacin, Vancomycin, Erythromycin & & 0 & 0 & $0 / 0$ \\
\hline
\end{tabular}




\section{Resistance and Sensitivity data of Staphylococcal species}

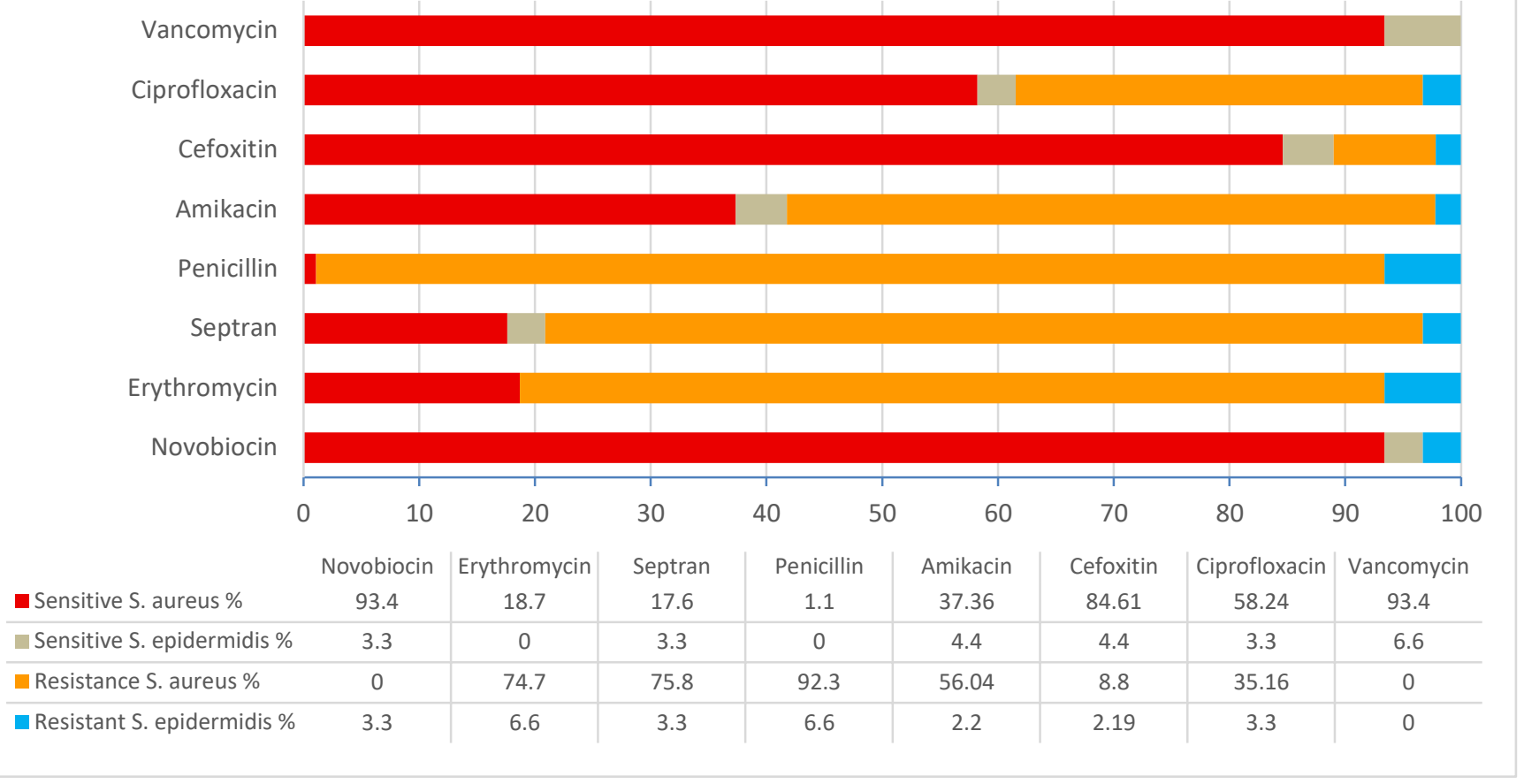

Fig. 4. Resistance and sensitivity data of Staphylococcus species to the tested antibiotics

Today, a large number of antibacterial combinations are available. Some combinations show similar behaviors to each other when assayed in vitro. Therefore, one drug can be tested to represent other similar or closely related compounds. In the present study, Staphylococcal species were tested against various antibiotics including cell wall synthesis inhibiting compounds and protein synthesis disrupting combinations, like erythromycin, trimethoprim/sulfamethoxazole, penicillin, ciprofloxacin, cefoxitin, vancomycin and two non-specific drugs, novobiocin, and amikacin. Today, methicillin-resistant S. aureus (MRSA) and S. epidermidis (MRSE) have emerged as a critical threat in both hospitals and communities [25]. Therefore, the samples were also tested to identify MRSA and MRSE. The compounds oxacillin or cefoxitin can be used as an alternative for all other $\beta$-lactams such as cephamycins), and susceptibility testing to these two drugs can predict resistance to all class of $\beta$-lactam antibiotics [26]. In a study, the cefoxitin disk diffusion test showed to be preferable to the oxacillin disk diffusion method for routine screening of MRSA. [27]. The bacteria species in the nasal samples showed the highest resistance to penicillin $(\approx 98.90 \%)$ followed by erythromycin $(81.32 \%)$. Previous studies have also reported $100 \%$ resistance of Staphylococcus species to penicillin and high rates of resistance to erythromycin [28]. The highest resistance rate to a single drug was observed for penicillin, followed by trimethoprim/sulfamethoxazole, erythromycin, ciprofloxacin, and cefoxitin. In the two-drug combinations, the highest resistance was observed to penicillin and trimethoprim/sulfamethoxazole (71 isolates), followed by penicillin and erythromycin (66 isolates), erythromycin and Trimethoprim/Sulfamethoxazole (52 isolates), penicillin and ciprofloxacin (29 isolates), ciprofloxacin and trimethoprim/sulfamethoxazole (25 isolates). In the three-drug combinations, the highest resistance was observed to the combination of penicillin, erythromycin, and trimethoprim/ sulfamethoxazole (61 isolates), followed by penicillin, ciprofloxacin, and trimethoprim/sulfamethoxazole (27 isolates), trimethoprim/sulfamethoxazole, erythromycin, and ciprofloxacin (25 isolates), and penicillin, erythromycin, and ciprofloxacin (25 isolates). In the four drugs combinations, the highest resistance was observed to erythromycin, penicillin, trimethoprim/sulfamethoxazole, and ciprofloxacin (23 isolates). Whereas, in the combination of five drugs, only 3 isolates were found to be resistant to the combination of trimethoprim/sulfame-thoxazole, penicillin, erythromycin, cefoxitin, and ciprofloxacin. All other combinations of five drugs were efficient against all Staphylococcal isolates (Table 2).

Resistance and sensitivity data of $S$. aureus and $S$. epidermidis indicated high resistance to penicillin in both species. High resistance to trimethoprim/sulfamethoxazole and erythromycin was also observed. The susceptibility to cefoxitin and ciprofloxacin among the $S$. aureus and $S$. epidermidis isolates was higher compared to penicillin, erythromycin, and trimethoprim. All species of Staphylococcus were found to be sensitive to vancomycin (Fig. 4).

Staphylococcal species, especially $S$. aureus are very prevalent in our community and are the primary cause of 
nosocomial and respiratory tract infections in children. In the current study, most of the isolates were identified to be $S$. aureus, and a smaller percentage were S. epidermidis. All the isolates were resistant to a minimum of 2 and a maximum of 5 antibacterial drugs. The MRSA was identified in $10 \%$ of the S. aureus isolates, whereas 33\% isolates were MRSE. Our results provide a better understanding of the epidemiology and determinants of Staphylococcal nasal colonization and can help public health authorities in Pakistan to adopt proper control and prevention measures.

\section{ACKNOWLEDGMENT}

The authors would like to acknowledge Higher Education Commission for funding the research under the grant NRPU-HEC 4269.

\section{CONFLICT OF INTEREST}

The authors declare that there are no conflicts of interest associated with this manuscript.

\section{REFERENCES}

1. Schito GC. The importance of the development of antibiotic resistance in Staphylococcus aureus. Clin Microbiol Inf. 2006; 12: 3-8.

2. Mika M, Mack I, Korten I, Qi W, Aebi S, Frey U, et al. Dynamics of the nasal microbiota in infancy: a prospective cohort study. J Allergy Clin Immunol. 2015; 135 (4): 905-12.

3. Rasmussen TT, Kirkeby LP, Poulsen K, Reinholdt J, Kilian M. Resident aerobic microbiota of the adult human nasal cavity. Apmis. 2000; 108 (10): 663-75.

4. Casadevall A, Pirofski LA. What is a host? Incorporating the Microbiota into the 'Damage-Response Framework'. Infect immunity. 2014; IAI-02627-14.

5. Tong SY, Davis JS, Eichenberger E, Holland TL, Fowler VG. Staphylococcus aureus infections: epidemiology, pathophysiology, clinical manifestations, and management. Clin Microbiol Rev. 2015; 28 (3): 603-61.

6. Verhoeven PO, Gagnaire J, Botelho-Nevers E, Grattard F, Carricajo A, Lucht F, et al. Detection and clinical relevance of Staphylococcus aureus nasal carriage: an update. Exp rev Antiinfective Ther. 2015; 12 (1): 75-89.

7. Stryjewski ME, Corey GR. Methicillin-resistant Staphylococcus aureus: an evolving pathogen. Clin Infec Dis. 2014; 58 (1): S10-S19.

8. Bassetti ME. Nicco E, Mikulska M. Why is communityassociated MRSA spreading across the world and how will it change clinical practice?" Int J Antimicrob Agents. 2009; 34 : S15-S19.

9. Von Eiff CK, Becker K, Machka K, Stammer H, Peters G. Nasal carriage as a source of Staphylococcus aureus bacteremia. New England J Med. 2001; 344 (1): 11-6.

10. Lindsay JA, Moore CE, Day NP, Peacock SJ, Witney AA, Stabler RA, et al. Microarrays reveal that each of the ten dominant lineages of Staphylococcus aureus has a unique combination of surface-associated and regulatory genes. J bacteriolo. 2006; 188 (2): 669-76.

11. Lowy FD. Antimicrobial resistance: the example of Staphylococcus aureus. The Journal of clinical investigation. 2003; 111 (9): 1265-73.

12. Gurusamy KS, Koti R, Toon CD, Wilson P, Davidson BR. Antibiotic therapy for the treatment of methicillin-resistant Staphylococcus aureus (MRSA) infections in surgical wounds. Cochrane Database Syst Rev. 2013; (8): CD009726-CD009726.

13. Klevens RM, Morrison MA, Nadle J, Petit S, Gershman K, Ray S, et al. Invasive methicillin-resistant Staphylococcus aureus infections in the United States. Jama. 2007; 298 (15): 1763-71.

14. Brown DF, Edwards DI, Hawkey PM, Morrison D, Ridgway GL, Towner KJ, et al. Guidelines for the laboratory diagnosis and susceptibility testing of methicillin-resistant Staphylococcus aureus (MRSA). J Antimicrob Chemother. 2005; 56 (6): 100018.

15. Martineau F, Picard FJ, Ke D, Paradis S, Roy PH, Ouellette $\mathrm{M}$, et al. Development of a PCR assay for identification of staphylococci at genus and species levels. J Clin Microbiol. 2001; 39 (7): 2541-7.

16. Jorgensen JH, Turnidge JD. Susceptibility test methods: dilution and disk diffusion methods. Manual of Clinical Microbiology. 11th Ed, American Society of Microbiology. $2015 ; 1253-73$.

17. Hudzicki J. Kirby-Bauer disk diffusion susceptibility test protocol. 2009.

18. Bauer AW, Perry DM, Kirby WM. Single-disk antibioticsensitivity testing of staphylococci: An analysis of technique and results. AMA Archives Interl Med. 1959; 104 (2): 208-16.

19. Wertheim HF, Melles DC, Vos MC, van Leeuwen W, van Belkum A, Verbrugh HA, et al. The role of nasal carriage in Staphylococcus aureus infections. The Lancet Infect Dis. 2005; 5 (12): 751-62.

20. Jourdain S, Smeesters PR, Denis O, Dramaix M, Sputael V, Malaviolle $X$, et al. Differences in nasopharyngeal bacterial carriage in preschool children from different socio-economic origins. Clin Microbiol Infect. 2011; 17 (6): 907-14.

21. Masuda K, Masuda R, Nishi JI, Tokuda K, Yoshinaga M, Miyata K. Incidences of nasopharyngeal colonization of respiratory bacterial pathogens in Japanese children attending day-care centers. Pediatr Int. 2002; 44 (4): 376-80.

22. Pathak A, Marothi Y, Iyer RV, Singh B, Sharma M, Eriksson B, et al. Nasal carriage and antimicrobial susceptibility of Staphylococcus aureus in healthy preschool children in Ujjain, India. BMC pediatr. 2010. 10 (1): 100.

23. Eibach D, Nagel M, Hogan B, Azuure C, Krumkamp R, Dekker D, et al. Nasal carriage of Staphylococcus aureus among children in the Ashanti Region of Ghana. PloS one. 2017; 12 (1): e0170320.

24. Lamaro-Cardoso J, De Lencastre H, Kipnis A, Pimenta FC, Oliveira LS, Oliveira RM, et al. Molecular epidemiology and risk factors for nasal carriage of Staphylococcus aureus and methicillin-resistant S. aureus in infants attending day care centers in Brazil. J Clin Microbiol. 2009; 47 (12): 3991-7. 
25. Boucher HW, Corey GR. Epidemiology of methicillinresistant Staphylococcus aureus. Clin Infect Dis. 2008; 46 (Suppl 5): S344-S349.

26. Magiorakos APA, Srinivasan, Carey RB, Carmeli Y, Falagas ME, Giske CG, et al. Multidrug-resistant, extensively drugresistant and pandrug-resistant bacteria: an international expert proposal for interim standard definitions for acquired resistance. Clin Microbiol Infect. 2012; 18 (3): 268-81.
27. Cauwelier BB, Gordts, Descheemaecker P, Van Landuyt H. Evaluation of a disk diffusion method with cefoxitin $(30 \mu \mathrm{g})$ for detection of methicillin-resistant Staphylococcus aureus. E J Clin Microbiol Infect Dis. 2004; 23 (5): 389-92.

28. Rağbetli C, Parlak M, Bayram Y, Guducuoglu H, Ceylan N. Evaluation of antimicrobial resistance in Staphylococcus aureus isolates by years. Interdis Perspect Infect Dis. 2016: 2016. 